\section{A cultured approach to ALS therapy}

\section{By Michael J. Haas, Associate Editor}

Although dysfunctional glial cells have long been implicated in amyotrophic lateral sclerosis progression, the precise mechanisms by which they contribute to the disease have remained poorly defined. Now, Harvard University researchers have identified a specific therapeutic target on glial cells-prostaglandin $\mathrm{D}_{2}$ receptor subtype DP1-and shown that reducing its expression can modestly prolong survival in a mouse model of the disease. ${ }^{1}$

Amyotrophic lateral sclerosis (ALS) is a progressive neurodegenerative disease that affects upper and lower motor neurons. This results in a range of symptoms that include muscle weakness and spasticity, loss of muscle function and difficulty in speaking, swallowing and breathing.

The only approved therapy is Sanofi's Rilutek riluzole, which slows damage to motor neurons by reducing glutamate neurotransmitter levels. However, even though the drug can prolong survival 3-5 months and may delay the need for tracheostomy or ventilator-assisted breathing, it does not improve overall function or quality of life.

The key challenge to the development of new ALS therapies is that the underlying disease biology is poorly understood. Although about $5 \%-10 \%$ of cases are linked to hereditary mutations in one of several genes, including superoxide dismutase 1 (SOD1) and chromosome 9 open reading frame 72 (C9orf72), ${ }^{2-5}$ it is not clear how these mutations cause the disease. Moreover, the majority of ALS cases are sporadic and have no known genetic cause.

Over the past two decades, studies in patients with ALS and mutant SOD1 animal models of the disease have demonstrated pathological roles for glial cells-including astrocytes, oligodendrocytes and microgliain the degeneration of motor neurons. ${ }^{6-9}$ These findings have led ALS researchers to propose that SOD1 mutations induce a gain of function in glial cells that renders them toxic to motor neurons. However, the mechanisms linking mutant SOD1 to glial cell toxicity remained elusive because it was difficult to dissect the complex functions of CNS and muscle cell types in vivo.

This changed about seven years ago, when the rapid development of mouse and human embryonic stem cell (hESC) technologies enabled the generation of SOD1-mutant glial cells that could be used for in vitro studies. ${ }^{10,11}$ A Harvard team led by Professor Kevin Eggan used this technology in cell coculture experiments to identify prostaglandin $\mathrm{D}_{2}$ $\left(\mathrm{PGD}_{2}\right)$ as a factor contributing to SOD1-mutant glial cell toxicity. ${ }^{11}$

However, the outstanding therapeutically relevant question was whether $\mathrm{PGD}_{2}$ was acting through one or both of its distinct receptors, DP1 (PTGDR1) or DP2 (PTGDR2; CRTH2; GPR44). Thus, Eggan and his team set out to determine which $\mathrm{PGD}_{2}$ receptor subtypes were contributing to glial toxicity and pin down which glial cell subtypes were responsible.

First, the team used cocultures of mutant SOD1 mouse glia and hESC-derived motor neurons to show that a DP1 inhibitor-but not a dual inhibitor of DP1 and DP2-decreased toxicity in the motor neurons compared with vehicle. Additional coculture experiments confirmed that signaling through DP1 on glial cells - not signaling through DP1 on motor neurons-was responsible for the previously observed toxic effect of $\mathrm{PGD}_{2}$. Knocking out one or both copies of DP1 in the glial cells also decreased toxicity compared with no alteration.

In mutant SOD1 mouse models of ALS, single or double DP1 knockout decreased disease progression and increased both motor neuron and animal survival compared with no alteration.

Lastly, the team's comparison of glial cell types from the mutant SOD1 mice and mutant SOD 1 and DP1 knockout mice showed that DP1 signaling in microglia-but not in astrocytes or other glial subtypes-was responsible for the toxic effect on motor -Deborah Eppstein, Q Therapeutics Inc. neurons. The team confirmed this by showing that a DP1 agonist induced toxicity in hESC-derived motor neurons cocultured with purified human fetal microglia but not in neurons cocultured with human fetal astrocytes.

Taken together, the findings suggest that inhibiting DP1 could prevent loss of motor neurons, thus slowing disease progression and extending the lifespan of patients with ALS. Results were published in Science Translational Medicine.

Eggan is an assistant professor of molecular and cellular biology at the Harvard Stem Cell Institute and an investigator at the Howard Hughes Medical Institute. His team included a researcher from the Leiden University Medical Center.

\section{Cellular cross-talk}

Companies contacted by SciBX said that Eggan's team had uncovered an important pathway that contributes to neuronal toxicity in ALS and suggested that DP1 inhibition-if it proves safe in the CNS - could be used in combination with other therapeutics in development, such as cell-based therapies.

"This is a very nice study that takes advantage of the ability to obtain purified populations of cells" for investigating disease mechanisms, said Mahendra Rao, cofounder, chief strategy officer and chair of the 
scientific advisory board at $\mathbf{Q}$ Therapeutics Inc. "The study also provides further confirmation of the role of glia and microglia in the etiology and pathology of ALS and the importance of modulating microglial function to treat the disease."

Daniel Anthony, an associate professor of pharmacology at the University of Oxford, agreed. "Astrocyte and microglial activation is a hallmark of ALS and correlates with disease progression, but it has been difficult to show whether the presence of glial cell-mediated inflammation can cause the death of neurons or is simply a result of the degenerative process," he said. "This study examines the contribution of DP1 activation to that process at the cellular levelhighlighting the role of microglial cells-and then in the whole animal to highlight DP1's clinical relevance."

However, Rao noted that although the team's findings demonstrate an important role for DP1 in microglial-mediated toxicity to motor neurons, "it is clearly not the whole picture, as suggested by the results of their experiments.”

Specifically, he pointed out that the team's coculture studies showed that $D P 1$ deficiency in mutant SOD1 glia resulted in full survival of the human motor neurons. But in the mutant SOD1 mouse models, single and double knockout of DP1 increased survival by only $8.2 \%$ and $6.7 \%$, respectively, compared with no alteration. "This implies that other mechanisms of toxicity are involved" besides DP1 signaling, he said.

Deborah Eppstein, president and CEO of Q Therapeutics, agreed. "We believe that therapeutic strategies using normal astrocytes or glial progenitors-such as our Q-cells, which can generate healthy astrocytes and oligodendrocytes-could result in broader protection of motor neurons than a compound that inhibits a single molecular target" such as DP1, she said. "This is because cells can respond to environmental cues to modulate their own functions and in turn modulate multiple molecular pathways" that support motor neuron growth, function and survival.

Nevertheless, Eppstein said, "because ALS is a very challenging disease that involves multiple pathways, down the road a combination of cellular therapy and compounds against single targets may provide synergistic benefits in treating ALS."

Q Therapeutics has Q-cells, human glial-restricted progenitor cells and their progeny, in preclinical development to treat ALS.

DP1 is known to be druggable. "Laropiprant, which has been used to treat flushing, is a selective DP1 blocker that seems to be well tolerated in humans," Anthony said.

In 2008, Merck \& Co Inc.'s Tredaptive, a combination of the selective DP1 inhibitor laropiprant and extended-release niacin, was marketed in multiple countries including in Europe to treat hypercholesterolemia and dyslipidemia. In the combination, DP1 reduced flushing caused by niacin-induced biosynthesis of the vasodilator $\mathrm{PGD}_{2}$. According to the EMA's 2008 Evaluations of Medicines for Human Use report on Tredaptive, preclinical studies showed that the drug had no CNS exposure.

In 2013, Merck withdrew Tredaptive from the market after a Phase III study showed that the drug plus statin therapy failed to reduce the incidence of cardiovascular events and also increased the incidence of nonfatal serious adverse events.
Merck declined SciBX's request to comment.

Anthony said that it is not clear whether inhibiting DP1 in the CNS would be desirable because $\mathrm{PGD}_{2}$-DP1's ligand-has shown neuroprotective effects in preclinical models of ischemia and excitotoxicity. "This is relevant because proposed mechanisms for cell death in ALS include glutamate excitotoxicity," which might therefore be exacerbated by inhibiting DP1.

Additionally, he noted that $\mathrm{PGD}_{2}$ is the most abundant prostanoid in the brain and its receptors are expressed by many neuronal tissuesmaking the long-term consequences of blocking DP1 in the CNS unclear. "For example, $\mathrm{PDG}_{2}$ induces sleep that is indistinguishable from normal physiological sleep, so one wonders whether DP1 inhibition might adversely affect circadian rhythms," he said. Thus, he wanted to see experiments in which the mutant SOD1 mouse models received the DP1 inhibitor over a long period of time to explore both potential benefits and side effects.

Eggan could not be reached for comment, but possible future studies could include determining whether glia harboring other ALSassociated gene mutations also induce toxicity in motor neurons via DP1 signaling.

According to the website for Harvard's Office of Technology Development, the university has filed a patent application covering the use of cocultures of mouse and hESC-derived human cells as in vitro models of ALS, and the IP is available for licensing.

Last year, Eggan and his colleague Lee Rubin, the Harvard Stem Cell Institute's director of translational medicine, partnered with Evotec AG to create induced pluripotent stem (iPS) cells from samples from patients with ALS to screen for and identify compounds with therapeutic potential. These also could be used to explore the mechanisms underlying motor neuron degeneration. ${ }^{12}$

At least two therapies directly targeting SOD1 are in preclinical development to treat ALS: Amorfix Life Sciences Ltd. and Biogen Idec Inc. have a mAb targeting misfolded SOD1, and Reata Pharmaceuticals Inc. has RTA 801, an oral SOD1 stabilizer.

Haas, M.J. SciBX 7(33); doi:10.1038/scibx.2014.974 Published online Aug. 28, 2014

\section{REFERENCES}

1. de Boer, A.S. et al. Sci. Transl. Med.; published online Aug. 6, 2014; doi:10.1126/scitranslmed.3009351

Contact: Kevin Eggan, Harvard University, Cambridge, Mass. e-mail: eggan@mcb.harvard.edu

Contact: A. Sophie de Boer, same affiliation as above e-mail: sdeboer@mcb.harvard.edu

2. Sareen, D. et al. Sci. Transl. Med. 5, 208ra149 (2013)

3. Donnelly, C.J. et al. Neuron $80,415-428$ (2013)

4. Lagier-Tourenne, C. et al. Proc. Natl. Acad. Sci. USA 110, E4530E4539 (2013)

5. Martz, L. SciBX 6(43); doi:10.1038/scibx.2013.1210

6. Boillée, S. et al. Neuron 52, 39-59 (2006)

7. Lepore, A.C. et al. PLoS ONE 6, e25968; published online Oct. 5, 2011; doi:10.1371/journal.pone.0025968

8. Lee, Y. et al. Nature 487, 443-448 (2012)

9. Kang, S.H. et al. Nat. Neurosci. 16, 571-579 (2013) 


\section{ANALYSIS}

\section{TARGETS \& MECHANISMS}

10. Di Giorgio, F.P. et al. Nat. Neurosci. 10, 608-614 (2007)

11. Di Giorgio, F.P. et al. Cell Stem Cell 3, 637-648 (2008)

12. Fishburn, C.S. SciBX 6(38); doi:10.1038/scibx.2013.1050

COMPANIES AND INSTITUTIONS MENTIONED

Amorfix Life Sciences Ltd. (TSX:AMF), Mississauga, Ontario,

Canada

Biogen Idec Inc. (NASDAQ:BIIB), Weston, Mass.

European Medicines Agency, London, U.K.

Evotec AG (Xetra:EVT), Hamburg, Germany
Harvard Stem Cell Institute, Cambridge, Mass.

Harvard University, Cambridge, Mass.

Howard Hughes Medical Institute, Chevy Chase, Md.

Leiden University Medical Center, Leiden, the Netherlands

Merck \& Co. Inc. (NYSE:MRK), Whitehouse Station, N.J.

Q Therapeutics Inc., Salt Lake City, Utah

Reata Pharmaceuticals Inc., Irving, Texas

Sanofi (Euronext:SAN; NYSE:SNY), Paris, France

University of Oxford, Oxford, U.K. 